\title{
Benign Maxillary Sinus Neoplasm
}

National Cancer Institute

\section{Source}

National Cancer Institute. Benign Maxillary Sinus Neoplasm. NCI Thesaurus. Code C4414.

A non-metastasizing neoplasm that arises from the maxillary sinus. 